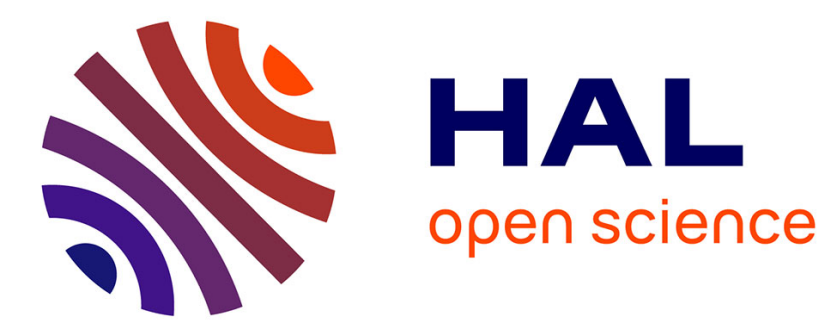

\title{
La sexualité des sujets transgenres et transsexuels saisie par les médias
}

Karine Espineira

\section{To cite this version:}

Karine Espineira. La sexualité des sujets transgenres et transsexuels saisie par les médias. Hermès, La Revue - Cognition, communication, politique, 2014, 69, p. 105-109. hal-01059100

\section{HAL Id: hal-01059100 https://hal.science/hal-01059100}

Submitted on 29 Aug 2014

HAL is a multi-disciplinary open access archive for the deposit and dissemination of scientific research documents, whether they are published or not. The documents may come from teaching and research institutions in France or abroad, or from public or private research centers.
L'archive ouverte pluridisciplinaire HAL, est destinée au dépôt et à la diffusion de documents scientifiques de niveau recherche, publiés ou non, émanant des établissements d'enseignement et de recherche français ou étrangers, des laboratoires publics ou privés. 
ESPINEIRA Karine, "La sexualité des sujets transgenres et transsexuels saisie par les médias », Karine Espineira, Revue Hermès, « Sexualités », Paris : Éditions CNRS, 2014, p. 105-109.

\author{
Karine Espineira \\ Université de Nice-Sophia Antipolis
}

\title{
La sexualité des sujets transgenres et transsexuels saisie par les médias
}

« Changer l'image des trans dans la société » est une préoccupation déclarée de longue date des groupes trans et des personnalités les plus médiatisées - préoccupation doublée, depuis les années 2000, de la volonté de « changer l'image des trans dans les médias ».

En se protégeant dans les médias, durant des décennies, de l'assimilation au cabaret, à la prostitution ou à la pornographie, les personnes s'auto-identifiant transsexuelles ${ }^{1}$ se sont ainsi défendues de toute forme de sexualité. Quant aux personnes se reconnaissant transgenres, elles ont connu une médiatisation plus tardive, confidentielle et polémique. Si les transsexuels semblent être relativement parvenus à se défaire de la sexualité, les personnes transgenres se sont vues dépossédées du genre et chargées de la sexualité ; ce qui semble avoir en partie invisibilisé leurs critiques de l'ordre des genres, de la binarité et du sexisme. Les modélisations médiatiques n'ont pas été sans effets sur le terrain, y générant confusions, divisions et concurrences.

Afin de mettre en lumière les processus croisés de sursexualisation et de désexualisation des identités trans, nous prendrons appui sur les résultats d'une observation participante menée de 2008 à 2012 (centrée sur les associations et collectifs transidentitaires) et sur l'étude de deux corpus audiovisuels ${ }^{2}$.

\section{Modalités trans de désignation et d'identification}

Le préfixe «trans » est associé au suffixe «identité » depuis les années 2000 dans le contexte français, après avoir été associé à la sexualité (transsexualité) depuis les années 1950 et au genre (transgenre) depuis les années 1970 dans un contexte international. Ainsi, le terme de «transidentité » est un terme parapluie dans lequel se reconnaissent des personnes transsexuelles et transgenres, ainsi que d'autres identités trans alternatives. Il cohabite avec les préfixes trans ou trans', l'usage de ces abréviations marquant le retournement de stigmate $^{3}$. Ces termes et préfixes produits par le monde associatif et par la militance transidentitaire sont désormais connus du grand public suite à la médiatisation croissante des parcours de vie des identités trans depuis les années 1970.

\footnotetext{
${ }^{1}$ Dans les discours médicaux et médiatiques, les personnes transsexuelles et transgenres sont distinguées par le fait que les premières auraient recours à l'opération de réassignation sexuelle tandis que les secondes n'en exprimeraient ni le désir ni le besoin.

${ }^{2}$ Le premier est constitué de 886 documents d'archives de l'Institut national de l'audiovisuel couvrant la période 1946-2010. Le second, annexe et documentaire, constitué de façon subjective, propose une centaine de références, essentiellement des productions étrangères.

${ }^{3}$ Réappropriation par une personne ou un collectif d'un terme ou d'une insulte la/le désignant sans son consentement. La réappropriation induit la transformation du sens, par le fait que celui ou celle qui le prononce n'est plus celui qui l'utilisait auparavant.
} 
Le public connait probablement moins la carte des positionnements identitaires que l'on peut dessiner à travers les acronymes et leurs débordements. L'acronyme MtF signifie male to female, traduit en français par «homme vers femme » (et non « mâle vers femelle »). Par FtM, il faut entendre female to male («femme vers homme »). Avec la politisation d'une partie de l'associatif transidentitaire, les acronymes ont été reformulés : ainsi, $\mathrm{Ft}^{*}$ ou FtX (plus rarement dans le sens $\mathrm{Mt}^{*}$ ) soulignent que le genre d'arrivée ou de destination est estimé inconnu ou indéterminé, l'astérisque ou le $\mathrm{X}$ marquant un refus d'assignation ${ }^{4}$. Les politiques transgenres sont lisibles et intelligibles par ces usages terminologiques et graphiques, usages pourtant méconnus et ignorés des médias.

La naissance du fait trans comme fait et question de société s'est accompagnée dans les médias de questionnements relatifs à l'identité, au genre et à la sexualité des personnes trans. Or, les personnes transsexuelles rejettent les termes les désignant parce qu'elles les jugent inadéquats: ils orientent sur la sexualité et non pas sur l'identité. Le terme «transsexuel » par exemple a été rejeté par Christine Jorgensen - consacrée première transsexuelle opérée de l'histoire, le $1^{\text {er }}$ décembre 1952, par la une du New York Daily News (vol. 34, n 136) : «Ex-GI Becomes Blonde Beauty». Avec Jorgensen, c'est le récit d'un premier changement de sexe qui se trouve médiatisé dans le monde entier, notamment à l'occasion de sa célèbre descente d'avion en 1953 à New York, lors de laquelle son hétérosexualité avait largement été mise en avant (Meyerowitz, 2006). Presque trente ans plus tard, le 19 octobre 1979, dans le Winnipeg Free Press, Jorgensen explique s'identifier comme trans-gender, terme qui lui semble plus apte à décrire son parcours.

En 1982, toujours dans une volonté affichée de dissocier identité et sexualité, elle illustre son propos : «le genre c'est ce que vous êtes » et la sexualité « ce qui regarde votre partenaire dans l'intimité ${ }^{2} »$. Les personnes identifiées et/ou auto-identifiées comme transsexuelles s'efforcent souvent de mettre en avant cette distinction dans les médias, et ce depuis les premiers débats en télévision et les premières vulgarisations du sujet par des représentants du corps médical ${ }^{6}$. Malgré cela, les intérêts croisés et divers pour la sexualité des personnes trans se lisent à travers la présomption/injonction d'hétérosexualité et les nombreuses spéculations sur la pratique de la sexualité des sujets non opérés.

\section{La sexualité des trans saisie par la télévision française}

Deux catégories principales de transidentités sont définies par opposition dans les imaginaires sociaux et médiatiques. La première est dite transsexuelle; son exposition dans les médias généraux depuis le milieu des années 1970 s'accompagne d'un appel à la tolérance et à l'acceptation. La seconde est dite travestie dans le contexte français jusqu'à ce que le terme transgenre soit connu. La télévision aborde très largement la thématique sous l'angle de la prostitution dite masculine ${ }^{7}$ liée à la pègre à Marseille, avant de parler d'une « prostitution trans » au bois de Boulogne dans le Paris des années 1980-1990.

La sexualité des personnes trans est un sujet de télévision, abordé plus ou moins directement. Elle est principalement présentée comme hétérosexuelle. Quand elle ne l'est pas, elle crée du trouble et de l'incompréhension. Il a été possible de le constater avec Ludwig

\footnotetext{
${ }^{4}$ Voir le témoignage de Vincent He-Say dans L'Ordre des mots, documentaire de Cynthia et Mélissa Arra (2007). Jamais télédiffusé, il est reconnu pour donner la parole à des identités non conformes politisées.

${ }^{5}$ « News From California: "Transgender" », Appeal Democrat, 11 mai 1982, p. A-10.

6 Quelques exemples: «La transsexualité », Aujourd'hui Magazine, Antenne 2, 27/09/1977; «Les transsexuels », Aujourd'hui madame, Antenne 2, 19/06/1980; «Plateau René Küss », JTNuit, Antenne 2, 29/06/1982 ; " D'un sexe à l'autre, elle ou lui ? », Les dossiers de l'écran, Antenne 2, 15/12/1987.

${ }^{7}$ Quelques exemples : «Prostitués », C'est à dire, Antenne 2, 25/02/1976; « De quoi avons-nous peur?», Vendredi, FR3, 17/06/1977; «Pour une dose d'enfer », Les mercredis de l'information, TF1, 15/12/1981; «P comme proxénète », Les mercredis de l'information, TF1, 09/06/1982.
} 
Trovato dans l'émission Ça se discute ${ }^{8}$ dont le portrait contraste avec celui, plus consensuel, de Stéphanie apparaissant au cours de ce même programme. Ludwig, qui se définit $\mathrm{Ft}^{*}$, parle sans retenue d'une sexualité libre ${ }^{9}$ tandis que Stéphanie donne les garanties d'une sexualité dite hétérosexuelle. Le couple qu'elle forme avec son ami attend l'opération pour vivre pleinement cet aspect de leur relation amoureuse. À quelques jours de cette étape importante, les discours disent une "asexualité d'acceptation» comme gage de bonnes mœurs. Elle semble obligatoire tant que l'ordre des genres n'est pas rétabli. Stéphanie et son compagnon sont-ils en mesure d'avouer une sexualité ? Doivent-ils être insoupçonnables ? Le transsexualisme semble bien marginaliser la sexualité (Hérault, 2010). Les gages à la normalité exigés par les protocoles mis en place par la médecine légale et reformulés par les médias ont été nombreux et se sont doublés de gages de moralité. L'asexualité est une défense car elle ne donne pas prise aux soupçons soit d'une sexualité débridée soit d'homosexualité. La désexualisation de l'identité vaut ainsi pour laissez-passer.

Un autre exemple peut être donné avec l'un des épisodes de la collection Tribunal ${ }^{10}$, qui met en scène le jugement d'une demande d'état civil de Jacqueline. Cette dernière a connu un parcours de vie proche des récits autobiographiques tel celui de Maud Marin (Le saut de l'ange, 1987). L'émission est documentée et illustre assez fidèlement le climat entourant les demandes de changement d'état civil. Le juge appelle le compagnon de Jacqueline à la barre : « Vous savez que génétiquement c'est un homme ? [...] Comment pouvez-vous aimer cette chose ? » On demande au compagnon de reconnaître qu'elle est "un homme » et non ce qu'elle dit être (une femme) - ce qui fait de lui un homosexuel. Les sanctions morales de ce type sont connues des personnes trans. Doit-on s'étonner alors des stratégies de défense mises en place?

Dans la catégorie «charme de l'ambiguïté », la figure médiatique trans est avant tout celle d'une femme dont on vante l'hyperféminité et le sex-appeal, dans les programmes s'intéressant au libertinage sous toutes ses formes ${ }^{11}$. Cette femme est dite "plus femme que les femmes » et laisserait planer le doute sur son entrejambe. Parle-t-on d'un travesti ou d'une transgenre du cabaret, de la prostitution, du monde du libertinage ? La sexualité des trans est aussi imaginée et/ou mise en scène dans l'imagerie pornographique. Celle-ci met en scène des "shemales ${ }^{12}$ » dans des productions que le numérique recycle sous une forme dite vintage dans les circuits de partage de fichiers peer to peer sur Internet. Notons que shemale est un terme péjoratif décrivant une personne sous hormones féminines (œstrogènes, etc.) mais qui n'a pas été opérée des parties génitales. Il désigne non seulement des personnes en situation de prostitution, mais aussi les actrices et modèles de films et revues de l'industrie pornographique. Le terme est d'autant plus offensant qu'appliqué à une personne transsexuelle (présumée opérée), il vaut - dans le contexte anglo-saxon notamment - pour accusation de prostitution. La prostitution est l'un des thèmes par lequel la sexualité des personnes trans a été saisie, hybridant fantasmes et voyeurismes, préventions et condamnations, comme l'illustre l'étude du pic de médiatisation du bois de Boulogne de la période 1988-1992 (Espineira, 2014).

\footnotetext{
${ }^{8}$ « Sexualité : comment assume-t-on son ambiguïté ? », Ça se discute, France 2, 20/10/2004.

${ }^{9}$ Voir son autoportrait dans le film Ludwig, France, 54 min., 2007.

${ }^{10}$ « Transsexuel », Tribunal, TF1, 07/11/1989.

11 Voir les séries documentaires : Sex in the world de Raphaëlle Bénisty, 4 volets de 52 minutes, Ladybirds Films, France, 2009. Sex in the world's cities 1 (6 volets) et Sex in the world's cities 2 (4 volets), collections dirigées par Marc Jappain, Ladybirds Films, France, 2010-2011. Le sujet trans est abordé dans plusieurs de ces volets.

12 Terme anglo-saxon qui s'orthographie aussi she-male; contraction de «elle » et «mâle ». Dans le langage populaire au XIX ${ }^{\mathrm{e}}$ siècle, le mot désigne une «femme agressive » s'imposant « comme un homme » (source : Online Etymology Dictionary).
} 
Enfin, dans le domaine de la pornographie, Internet et ses tubes (Perea, 2012) professionnels et amateurs, comme les productions cinématographiques, donnent lieu à d'autres représentations. Le TGirl Sex, qui joue sur ces ressorts connus de la femme trans non opérée, est désormais un genre à part entière dans son aspect «massif et stéréotypé » (RonanDubois, 2013) dans lequel la femme trans se voit déclinée en tranny ou en transsexual, qu'elle soit baby-sitter, cheerleader, prostitute ou escort, young Asian tranny comme ladyboy. Elle est proie/chasseuse, passive/active ou encore dominatrice. La tranny ou la Tgirl ne se contente pas d'être une figure sexuelle multiculturelle, elle associe et hybride figures transgenres et transsexuelles. Les productions pornographiques de la «raillerie des conventions de genre » (Boucher, 2013), de la "résistance » et du post-porno trans "doté d'un contenu politique » (Borghi, 2013) se retrouvent dans d'autres circuits avec les productions de Morty Diamond, Tobi Hill-Meyer ou encore de Buck Angel qui rompent avec un porno centré sur les sujets trans à usage d'un public non trans). Si l'industrie pornographique sursexualise le sujet trans, la pornographie produite et diffusée par des personnes trans introduit une confusion des genres, ne devant pas être comprise uniquement au sens de trouble mais aussi entendue comme rupture avec les déterminismes biologiques.

\section{Processus croisés liés à la sexualité et au genre}

En prêtant aux personnes transgenres une paraphilie ou encore l'autogynéphilie ${ }^{13}$ (Blanchard, 1989), la sexualisation de la condition trans semble très accentuée. Au contraire, le traitement du transsexualisme donne lieu à une "focalisation consensuelle sur le genre " (Hérault, 2010) tout en exigeant des personnes les preuves d'une féminité ou d'une masculinité atteignant souvent à l'archétype (Thomas, 2007). La femme dite transsexuelle a dû et doit être encore une personne morale sur la scène médiatique comme sur la scène du réel. Sa quête d'identité est un fait de société, disent les médias. Le changement de sexe est accordé sous la condition d'une adhésion totale à l'ordre binaire des genres. La femme transgenre est quant à elle sursexualisée par association aux représentations de la pornographie et assimilée à la prostitution. Nous assistons à un processus de dégenration par focalisation sur une sexualité dite déviante.

Les mécanismes de désexualisation et de sursexualisation des représentations sociales et médiatiques des personnes trans se laissent approcher par les témoignages de vie, doublés des diagnostics médicaux et psychiatriques. On plaide pour une antécédence du genre chez les transsexuels : le sexe (l'organe) devient un construit pour réparer «l'erreur de la nature ». Le sujet semble devoir être désexualisé pour être acceptable et se voir genré. Pour qualifier les transgenres et les travestis, les commentaires disent par contre une antécédence présumée du « sexe " au sens de sexualité et non de genre social : la pratique de la sexualité prime sur le genre vécu et relègue les personnes au statut de « fausses femmes » par exemple.

Deux représentations sociales et médiatiques des personnes trans semblent ainsi cohabiter dans l'espace social et médiatique mais n'y ont pas la même valeur. Le processus de surgenration et de désexualisation rend le sujet transsexuel moralement plus acceptable tandis que la sursexualisation et la « dégenration » du sujet transgenre le cantonnent à la marge. Les représentations médiatiques semblent alors laisser affleurer les sanctions sociales de « franchissement de genre ».

\section{NOTES}

\footnotetext{
${ }^{13}$ La paraphilie désigne des pratiques sexuelles considérées comme déviantes, tandis que l'autogynéphilie désigne l'excitation sexuelle ressentie par un homme à s'imaginer en femme.
} 


\section{RÉFÉRENCES BIBLIOGRAPHIQUES}

Borghi, R., « Post-Porn », Rue Descartes, n 79, 2013, p. 29-41.

BLANCHARD, R., "The concept of autogynephilia and the typology of male gender dysphoria », The Journal of Nervous and Mental Disease, 177, 1989, p. 616-623.

BOUCHER, P., « Le cri de la hyène : trans, cybermedia et post-pornographie », Rue Descartes, $\mathrm{n}^{\circ}$ 79, 2013, p. 16-28.

ESPINEIRA, K., «La médiatisation des politiques transgenres : du statut de contre-public à l'inégalité de la représentation ", Revue française des sciences de l'information et de la communication [en ligne], $\mathrm{n}^{\circ} 4,1^{\text {er }}$ janv. 2014. En ligne sur : <rfsic.revues.org/695>, consulté le $07 / 04 / 2014$.

ESPINEIRA, K., «Transidentités et média(tion)s », in RAIBAUd, Y. et AlessandRIn, A. (dir.), Géographie des homophobies, Paris, Armand Colin, 2013, p. 57-69.

HÉRAut, L., "Usages de la sexualité dans la clinique du transsexualisme », L'Autre. Cliniques, cultures et sociétés, vol. 11, n 3, 2010, p. 278-291.

Meyerowitz, J., How Sex Changed. A History of Transsexuality in the United States, Cambridge, Harvard University Press, 2002.

Meyerowitz, J., "Transforming Sex: Christine Jorgensen in the Postwar U.S. », OAH Magazine of History, vol. 20, $\mathrm{n}^{\circ} 2$, mars 2006, p. 16-20.

PARKer, J., «Christine Recalls Life as Boy from the Bronx », Newsday/Winnipeg Free Press, 18 octobre 1979.

PEREA, F., «Les sites pornographiques par le menu: pornotypes linguistiques et procédés médiatiques », Genre, sexualité \& société [en ligne], $\mathrm{n}^{\circ} 7,1^{\mathrm{er}}$ juin 2012. En ligne sur: <gss.revues.org/2395>, consulté le 07/04/2014.

RONAN-DuBOIS, F., « La cohérence visuelle et formelle des tubes pornographiques. Le cas de la catégorie asian sur un tube gay », Revue Proteus, $\mathrm{n}^{\circ}$ 5, dossier "Pornographies : entre l'animal et la machine », 2013, p. 48-54.

ThOMAS, M.-Y., «La controverse trans », Mouvements, dossier «Trans Révolution» [en ligne], 5 oct. 2007. En ligne sur: <www.mouvements.info/La-Controverse-trans.html>, consulté le 07/04/2014. 